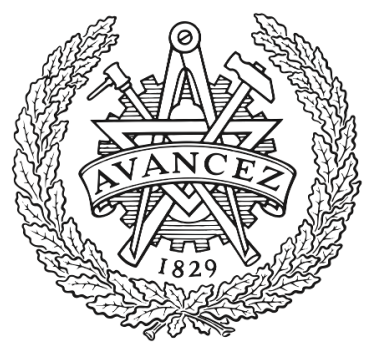

CHALMERS

UNIVERSITY OF TECHNOLOGY

\title{
PM-64QAM Coherent Optical Communications Using a Dark-Pulse Microresonator Frequency Comb
}

Downloaded from: https://research.chalmers.se, 2023-04-26 08:07 UTC

Citation for the original published paper (version of record):

Mazur, M., Lorences Riesgo, A., Fülöp, A. et al (2018). PM-64QAM Coherent Optical

Communications Using a Dark-Pulse Microresonator Frequency Comb. Conference on Lasers and Electro-Optics, CLEO 2018. http://dx.doi.org/10.1364/CLEO_SI.2018.SW3C.1

N.B. When citing this work, cite the original published paper. 


\title{
PM-64QAM Coherent Optical Communications Using a Dark-Pulse Microresonator Frequency Comb
}

\author{
Attila Fülöp $^{1 *}$, Mikael Mazur ${ }^{1}$, Abel Lorences-Riesgo ${ }^{1, T}$, Pei-Hsun Wang ${ }^{2}$, Yi Xuan ${ }^{2,3}$, Dan. E. Leaird ${ }^{2}$, \\ Minghao $\mathbf{Q i}^{2,3}$, Peter A. Andrekson ${ }^{1}$, Andrew M. Weiner ${ }^{2,3}$, and Victor Torres-Company ${ }^{1}$ \\ ${ }^{1}$ Photonics Laboratory, Department of Microtechnology and Nanoscience, Chalmers University of Technology, SE-41296 Göteborg, Sweden \\ ${ }^{2}$ School of Electrical and Computer Engineering, Purdue University, West Lafayette, IN 47907-2035, USA \\ ${ }^{3}$ Birck Nanotechnology Center, Purdue University, West Lafayette, IN 47907-2035, USA \\ ${ }^{T}$ Now at IT-Instituto de Telecomunicações, 3810-193 Aveiro, Portugal \\ *attila.fulop@chalmers.se
}

\begin{abstract}
Dark-pulse microresonator combs exhibit efficient pump-to-comb power conversion. Using on-chip pump powers of $21 \mathrm{dBm}$, we show 20-channel PM-64QAM-based data transmission. These results represent the highest-order modulation format encoded onto any integrated comb. OCIS codes: (060.1660) Coherent communications; (190.4390) Nonlinear optics, integrated optics
\end{abstract}

\section{Introduction}

Microresonator frequency combs are generated by utilizing the Kerr effect in a nonlinear optical cavity [1]. The resonator can be designed so the spacing between the equidistant lines is on the order of tens of $\mathrm{GHz}$, making it suitable for optical communications [2]. Recent experiments with silicon nitride-based combs have shown that they can operate in several multi-wavelength scenarios [3-6]. These devices could enable future chip-scale transceivers by allowing co-integration of the comb source with modulators and other components on a single chip [7].

Recently, a new class of mode-locked microresonator combs, consisting of circulating dark pulses has been demonstrated [8]. These comb states display high pump-to-comb power conversion efficiencies [9]. This is of significant interest in the context of optical communications as it can allow for chip-scale comb sources requiring only moderate pump powers. Here we present the first dark-pulse comb transmission experiment. The high conversion efficiency allowed us to reach the highest-order modulation format used with any integrated comb source to date. We performed $80 \mathrm{~km}$ transmission with 20 parallel data channels using $20 \mathrm{GBd}$ PM-64QAM. The on-chip pump power was about $21 \mathrm{dBm}$, a level compatible with state-of-the-art chip-scale laser sources [10]. This shows the potential for dark-pulse combs as light sources in fully integrated multi-wavelength transceivers.

\section{Comb generation}

The optical frequency comb shown in Fig. 1(a) was generated by pumping a high-Q ( $>1$ million) silicon nitride microresonator [11] with a sub-10 kHz linewidth continuous-wave pump laser amplified to $25.6 \mathrm{dBm}$. We estimated the coupling losses to be $5 \mathrm{~dB}$ per facet, yielding an on-chip pump-to-comb conversion efficiency above $20 \%$ (as defined in [5]). To initialize the comb state, the pump laser was tuned from the lower wavelength side into a cavity resonance located at $1540 \mathrm{~nm}$. Stable operation of the comb state over the measurement time was ensured by monitoring the power in the comb line at $1536 \mathrm{~nm}$. That power was then used as feedback for adjusting the pump laser wavelength [12]. The resulting $230 \mathrm{GHz}$ comb line spacing was set by the $100 \mu \mathrm{m}$ ring radius. To verify the dark-pulse comb state, the time-domain waveform was measured at the resonator's drop port using a $500 \mathrm{GHz}$ optical oscilloscope. The result is shown in Fig. 1(b). As the system's drop port was only weakly coupled to the ring, the through port was used instead for the data transmission experiment.
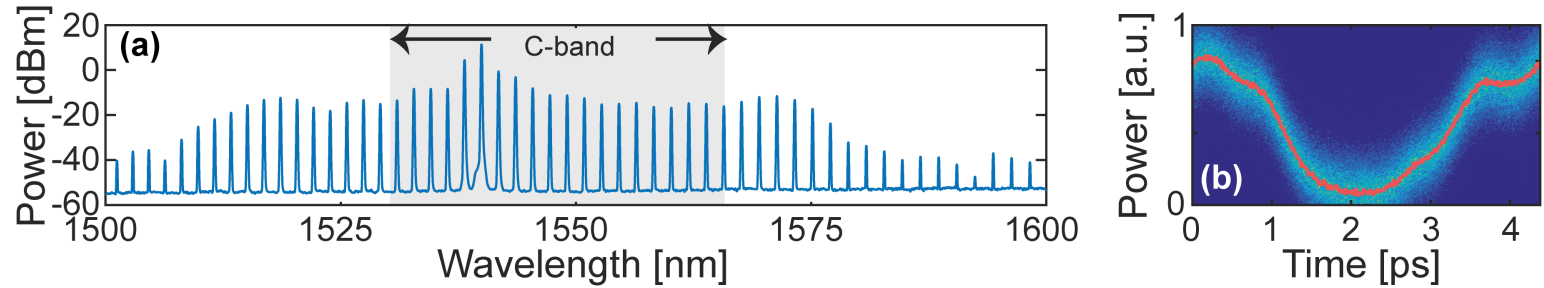

Fig. 1. (a) Optical spectrum (0.1 nm RBW) of the dark-pulse frequency comb used for the data transmission as seen at the output of the chip's through port. (b) The power of the intracavity waveform measured at the device's drop port.

\section{Data transmission}

The high power-per-line in the comb enables the usage of high-order coherent modulation formats. To demonstrate this in a system experiment, we modulated data onto $20 \mathrm{comb}$ lines within the C-band (between $1531 \mathrm{~nm}$ and 
$1566 \mathrm{~nm}$ ). The setup is shown in Fig. 2(a). Splitting the comb lines into two arms before modulation allowed sending independent data on neighboring channels and minimizing the number of carriers that were fed to a single modulator. The two random 20 GBd 64QAM data streams were generated using an arbitrary waveform generator (AWG) with a sequence length of $2^{16}$ symbols. To emulate a dual-polarized transmitter, a polarization-multiplexing stage with a $1 \mathrm{~m}$ (about 100 symbols) long decorrelation arm was included. The modulated carriers were then transmitted through an $80 \mathrm{~km}$ fiber link with all channels having above $33 \mathrm{~dB}$ OSNR (at $0.1 \mathrm{~nm}$ bandwidth).

A dual-polarized coherent receiver was used to receive each data channel individually. We used a tunable external-cavity laser (with $<100 \mathrm{kHz}$ linewidth) as local oscillator. The waveforms were recorded using a $23 \mathrm{GHz}$ bandwidth real-time oscilloscope operated at $50 \mathrm{GS} / \mathrm{s}$. The transmitted data symbols were then recovered offline using standard digital signal processing algorithms compensating for receiver, link and transmitter impairments. More than 9 million bits were decoded in each dual-polarized channel to calculate the received bit error ratio (BER). The results are shown in Fig. 2(b) with example received constellations displayed for both polarizations for the channel located at $1531 \mathrm{~nm}$ in Fig. 2(c). All channels had received BERs below 7 $10^{-3}$ allowing for the usage of hard-decision forward error correcting codes with $9.1 \%$ overhead [13]. The final aggregate data rate was $4.4 \mathrm{~Tb} / \mathrm{s}$.
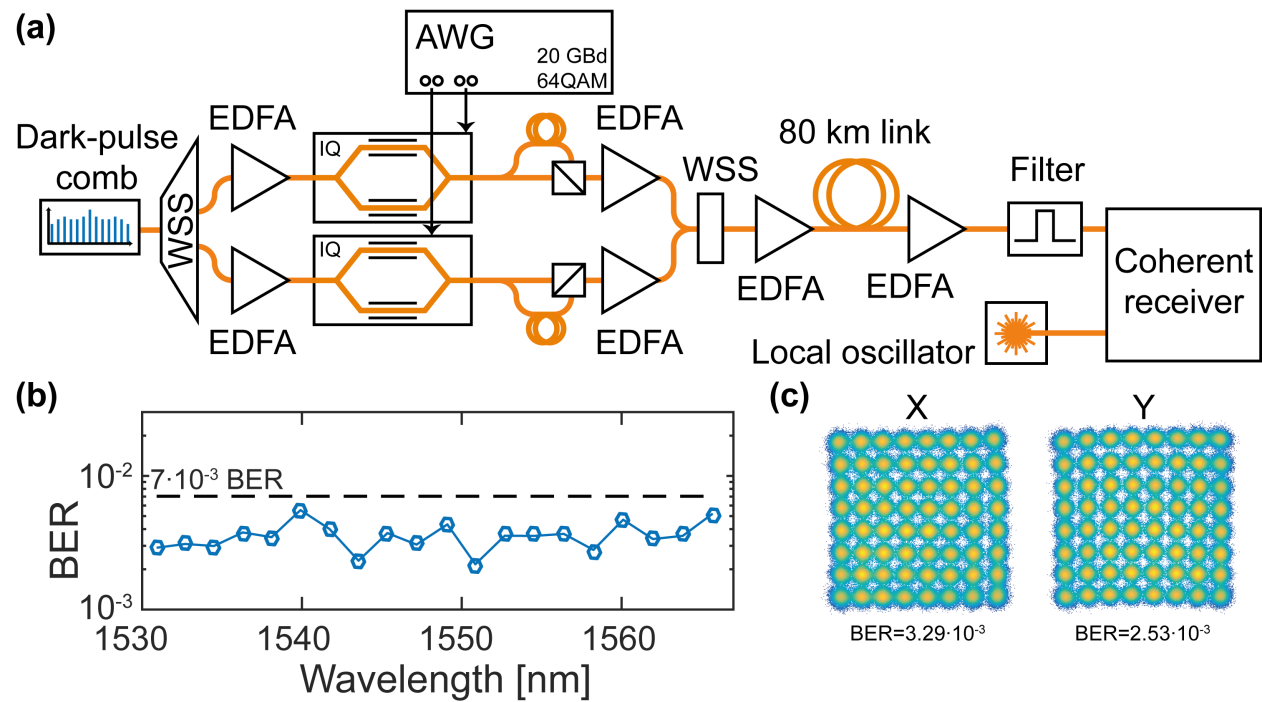

(c)

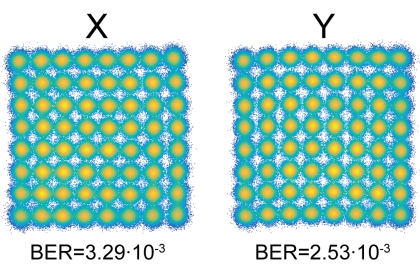

Fig. 2. (a) Schematic of the system setup. Two wavelength-selective switches (WSSs) were used to spectrally flatten the comb and compensate for the gain spectrum of the erbium-doped fiber amplifiers (EDFAs). The 20 GBd 64QAM data signal was generated using an arbitrary waveform generator (AWG). (b) Resulting bit-error ratios (BERs) for all 20 data channels allowing for error-free transmission using a hard-decision FEC [13]. (c) Received constellation diagrams and BERs for both polarizations for the channel at $1531 \mathrm{~nm}$.

\section{Conclusions}

We have shown the first coherent data transmission experiment performed with a dark-pulse microresonator comb source as well as the highest-order data modulation encoded onto any integrated comb source. The off-chip pump power was kept below $26 \mathrm{dBm}$ with an estimated on-chip pump level of $21 \mathrm{dBm}$. We have shown that dark-pulse combs are simultaneously compatible with high-order modulation formats and pump powers within reach of chipscale lasers. This makes them an attractive light source for future chip-scale multi-wavelength transceivers.

\section{References}

[1] P. Del'Haye et al., "Optical frequency comb generation from a monolithic microresonator," Nature 450, 1214 (2007).

[2] J. S. Levy et al., "CMOS-compatible multiple-wavelength oscillator for on-chip optical interconnects," Nat. Photonics 4, 37 (2010).

[3] J. Pfeifle et al., "Coherent terabit communications with microresonator Kerr frequency combs," Nat. Photonics 8, 375 (2014).

[4] P. Marin-Palomo et al., "Microresonator-based solitons for massively parallel coherent optical communications," Nature 546, 274 (2017).

[5] A. Fülöp et al., "Long-haul coherent communications using microresonator-based frequency combs," Opt. Express 25, 26678 (2017).

[6] C. Bao et al., "Tunable insertion of multiple lines into a Kerr frequency comb using electro-optical modulators," Opt. Lett. 42, 3765 (2017).

[7] W. D. Sacher et al., "Multilayer Silicon Nitride-on-Silicon Integrated Photonic Platform for 3D ...," in CLEO, (OSA, 2016$)$, pp. JTh4C.3.

[8] X. Xue et al., "Mode-locked dark pulse Kerr combs in normal-dispersion microresonators," Nat. Photonics 9, 594 (2015).

[9] X. Xue et al., "Microresonator Kerr frequency combs with high conversion efficiency," Laser Photon. Rev. 11, 1600276 (2017).

[10]N. Kobayashi et al., "Silicon Photonic Hybrid Ring-Filter External Cavity Wavelength Tunable Lasers," J. Light. Technol. 33, 1241 (2015).

[11]Y. Xuan et al., "High-Q silicon nitride microresonators exhibiting low-power frequency comb initiation," Optica 3, 1171 (2016).

[12] A. Fülöp et al., "Active feedback stabilization of normal-dispersion microresonator combs," in CLEO Eur., (IEEE, 2017), pp. CD-P.45.

[13]L. M. Zhang and F. R. Kschischang, "Staircase Codes With 6\% to 33\% Overhead," J. Light. Technol. 32, 1999 (2014).

We acknowledge support from the Swedish Research Council (VR), the NSF, DARPA, and AFOSR. 